\title{
TEMPO DE PERMANÊNCIA E MOTIVOS DE RETIRADA DO CATETER VENOSO CENTRAL DE PACIENTES RENAIS CRÔNICOS EM HEMODIÁLISE AMBULATORIAL
}

\author{
DURATION OF USE AND REASONS FOR REMOVAL \\ OF CENTRAL VENOUS CATHETER IN PATIENTS \\ WITH CHRONIC KIDNEY DISEASE UNDERGOING \\ AMBULATORY HEMODIALYSIS
}

\author{
Karen Ferreira dos Santos ${ }^{1}$, Guilherme Breitsameter ${ }^{2}$, \\ Maria Conceição da Costa Proença ${ }^{3}$, Fernanda Guarilha Boni ${ }^{4}$, \\ Isabel Cristina Echer ${ }^{5}$
}

Clin Biomed Res. 2021:41(1):12-17

1 Hospital da Brigada Militar de Porto Alegre, Universidade Federal do Rio Grande do Sul (UFRGS). Porto Alegre, RS, Brasil.

2 Hospital de Clínicas de Porto Alegre, Universidade Federal do Rio Grande do Sul (UFRGS). Porto Alegre, RS, Brasil.

3 Hospital de Clínicas de Porto Alegre, Universidade Federal do Rio Grande do Sul (UFRGS). Porto Alegre, RS, Brasil.

4 Escola de Enfermagem, Universidade Federal do Rio Grande do Sul (UFRGS). Porto Alegre, RS, Brasil.

5 Departamento de Assistência e Orientação Profissional da Escola de Enfermagem, Universidade Federal do Rio Grande do Sul (UFRGS). Porto Alegre, RS, Brasil.

Autor correspondente: Karen Ferreira dos Santos karendosantos@gmail.com Hospital da Brigada Militar de Porto Alegre, Universidade Federal do Rio Grande do Sul (UFRGS)

Rua Allan Kardec, 637 94856-010, Alvorada, RS, Brasil.

\section{RESUMO}

Introdução: A permanência prolongada de Cateter Venoso Central (CVC) em Hemodiálise (HD) está relacionado a maior risco de complicações. O objetivo deste estudo foi avaliar o tempo, em dias, de permanência e o motivo de retirada do CVC em pacientes renais crônicos submetidos à HD ambulatorial em um seguimento de 10 meses.

Métodos: Estudo longitudinal, retrospectivo, realizado em unidade de HD de um hospital público no sul do Brasil, no período de janeiro a setembro/2019. A coleta de dados foi realizada pelos pesquisadores a partir de prontuário eletrônico e planilha de registros dos doentes renais crônicos em hemodiálise por CVC de curta e longa permanência no período em estudo. Projeto aprovado no Comitê de Ética em Pesquisa da instituição.

Resultados: Foram avaliados 91 cateteres de 55 pacientes, com prevalência do sexo feminino $33(60 \%)$, média de idade $55 \pm 18$ anos e cor branca $42(76,3 \%)$. Quarenta e sete (52\%) dos CVCs eram de curta permanência, e 70 (76,9\%) inseridos em veia jugular direita. O tempo de hemodiálise com CVCs de curta permanência variou de quatro a 190 dias com mediana de 47 dias $(21,7-69,3)$ e os de longa permanência de 47 a 1.486 dias, com mediana de 231 (95-676). O principal motivo de retirada dos cateteres foi a troca por outro CVC 17 (36,2\%). A taxa de suspeita e/ou infecção foi de $14(15,4 \%)$.

Conclusão: O tempo de permanência dos CVC foi prolongado o que expõe os pacientes a riscos. O principal motivo para retirar o CVC não foi a confecção de Fístula Arteriovenosa (FAV), apontando para a necessidade de revisar os processos assistenciais visando modificar e priorizar essa prática.

Palavras-chave: Cateteres venosos centrais; fístula arteriovenosa; diálise renal; infecção

\section{ABSTRACT}

Introduction: The prolonged permanence of Central Venous Catheter (CVC) in Hemodialysis (HD) is related to an increased risk of complications. The aim of this study was to evaluate duration of use, in days, and reasons for removal of central venous catheter (CVC) in patients with chronic kidney disease undergoing ambulatory hemodialysis in a 10-month follow-up.

Methods: This longitudinal, retrospective study was conducted at the hemodialysis unit of a public hospital in southern Brazil, from January to September 2019. Data collection was performed by the researchers using electronic medical records and a spreadsheet of records of patients with chronic kidney disease undergoing hemodialysis by short- and long-term CVC in the study period. The project was approved by the institution's Research Ethics Committee. 
Results: Ninety-one catheters from 55 patients were evaluated. Thirty-three participants were female (60\%), mean age was $55 \pm 18$ years, and 42 were white (76.3\%). Forty-seven (52\%) CVCs were short-term, and 70 (76.9\%) CVCs were inserted in the right jugular vein. Hemodialysis duration with short-term CVCs ranged from four to 190 days with a median of 47 (21.7-69.3) days, and long-term CVCs ranged from 47 to 1486 days with a median of 231 (95-676) days. The main reason for removing the catheter was replacing it with another CVC, in 17 cases $(36.2 \%)$. The number of cases of infection or suspected infection was 14 (15.4\%).

Conclusions: The duration of CVC use was prolonged, which exposes patients to risks. The main reason for removing the CVC was not related to creating an arteriovenous fistula, demonstrating the need to review the care processes aiming to modify and prioritize this practice.

Keywords: Central venous catheters; arteriovenous fistula; renal dialysis; infection

\section{INTRODUÇÃO}

O Cateter Venoso Central (CVC) é um acesso vascular eficiente que permite o uso imediato e tem benefícios em casos específicos como nas urgências dialíticas. No entanto, nos pacientes em diálise pode estar relacionado a eventos adversos como fluxo sanguíneo inadequado, manipulação frequente, tração, possíveis falhas na assepsia, tanto por parte da equipe de saúde quanto do próprio paciente ${ }^{1-3}$.

Considera-se a Fístula Arteriovenosa (FAV) o acesso mais indicado para realizar o tratamento hemodialítico, devido a fluidez de uma artéria diretamente para uma veia, o que proporciona um fluxo sanguíneo adequado e regular, além de ser o procedimento mais seguro para o paciente ${ }^{4}$. Sendo assim, a terapia hemodialítica em Doença Renal Crônica (DRC) somente deve ocorrer por CVC quando, por algum motivo específico, não puder acontecer por FAV, como por exemplo, urgências, necessidade de repouso do peritônio, rejeição aguda de transplante, e alterações na FAV ${ }^{3}$.

Caso haja necessidade da permanência de cateter por tempo maior que duas semanas ou se o paciente não possuir outras opções de acesso vascular, recomenda-se o uso de CVC de longa permanência, o qual é tunelizado e possui cuff que proporciona barreira protetora e menor risco de infecção ${ }^{4,5}$. Cabe ressaltar que o uso de CVC de longa permanência traz limitações à vida do indivíduo, pois existe necessidade de cuidados específicos para prevenir tração e infecção, assim como, reduz a qualidade da hemodiálise e aumenta a mortalidade ${ }^{3,6}$.

Conforme os guidelines, não há limite de tempo máximo para o uso do cateter de longa permanência, mas é necessária uma avaliação regular para determinar se o CVC continua sendo o acesso de diálise mais apropriado para cada paciente $e^{5,7}$. Contudo, observase um número elevado de pacientes em hemodiálise por CVC sem contraindicação de FAV. Além disso, a literatura aponta que o tempo de permanência desses acessos são elevados, expondo o paciente a maior período aos riscos relacionados à presença do cateter ${ }^{6,8}$.
O risco de adquirir infecção é diretamente proporcional ao período em que o paciente permanece com CVC, sendo esse o motivo de troca ou retirada de cateteres na maioria dos casos. Dentre os demais motivos para retirar o CVC estão a perda de funcionalidade, insuficiência renal aguda revertida, início em diálise peritoneal, óbito e maturação da $\mathrm{FAV}^{6,9}$.

Nesta perspectiva, acompanhar os pacientes crônicos ambulatoriais em hemodiálise pelo CVC de curta ou longa permanência se faz necessário para melhor compreender essa realidade. Assim, este estudo tem o objetivo avaliar o tempo, em dias, de permanência e o motivo de retirada de CVC em pacientes renais crônicos submetidos à hemodiálise ambulatorial, em um seguimento de dez meses.

\section{MÉTODOS}

Trata-se de estudo longitudinal, retrospectivo do uso CVC de curta e longa permanência em pacientes renais crônicos submetidos à hemodiálise de ambulatório, em um segmento de 10 meses. A pesquisa foi realizada em um serviço de hemodiálise de um hospital público e universitário do sul do Brasil, que oferece ampla assistência ao paciente com comprometimento renal crônico ou agudo nas situações de tratamento conservador, dialítico e transplante renal. A unidade realiza mensalmente em média 708 sessões de hemodiálise ambulatorial, destas, aproximadamente $40 \%$ são realizadas por CVC de curta ou longa permanência.

A população foi composta por pacientes portadores de DRC que realizaram tratamento hemodialítico ambulatorial no referido hospital. A amostra foi por conveniência e incluiu todos os doentes renais crônicos que realizavam hemodiálise, três vezes por semana, por meio de CVC no período de janeiro a setembro de 2019. Foram excluídos os pacientes em diálise peritoneal, hemodiálise por FAV, ou doentes renais agudos que dialisaram apenas durante o período da internação hospitalar.

Todos os pacientes da amostra tiveram seus cateteres implantados por médico nefrologista no hospital campo deste estudo, sob técnica asséptica. 
A partir da segunda sessão de hemodiálise com o CVC, o local da inserção foi protegido com curativo transparente conforme protocolo institucional. Ao término de cada sessão de hemodiálise, os lúmens dos CVCs foram preenchidos por solução de heparina $5.000 \mathrm{u} / \mathrm{ml}$, com o volume indicado no próprio cateter pelo fabricante.

A coleta de dados ocorreu no período de setembro a outubro de 2019 , foi realizada pelos pesquisadores por meio de busca dos dados em prontuário eletrônico e planilha específica para os cateteres inseridos, disponível na unidade em estudo. Inicialmente, foram coletadas as informações constantes nesta planilha que é atualizada diariamente pelos enfermeiros e contém dados referentes à identificação do paciente, data do implante do cateter, local de inserção, tipo de CVC, data e motivos de retirada.

Os dados sociodemográficos, clínicos e tempo em hemodiálise foram coletados do prontuário eletrônico. As variáveis coletadas foram: idade, sexo, cor, escolaridade, diagnóstico de diabetes e/ou Hipertensão Arterial Sistêmica (HAS), tempo em hemodiálise, data, tipo e local da inserção de CVC, tempo de permanência com cateter e motivos de retirada.

Os dados foram organizados e codificados em banco de dados criado no programa Microsoft Excel $\mathbb{R}$ e após foram realizadas análises descritivas pelo software Statistical Package for the Social Sciences (SPSS) versão 23.0. As variáveis contínuas foram descritas por média e desvio padrão para aquelas com distribuição normal ou mediana e intervalo interquartil para as assimétricas.

Este estudo foi aprovado pelo Comitê de Ética em Pesquisa da instituição e o número do Certificado de Apresentação de Apreciação Ética (CAAE) foi 86412518900005327 . Os pesquisadores assinaram o termo de comprometimento para utilização de dados de prontuário.

\section{RESULTADOS}

Foram avaliados 91 cateteres, de 55 pacientes, totalizando 6.022 sessões de hemodiálise, com frequência variando de duas a 1.287 no período estudado, com mediana de $55(33,5-123,5)$ sessões. O tempo em que esses pacientes estavam em hemodiálise oscilou de 14 dias a 19 anos, com mediana de 290 dias (124-446).

Houve predominância, do sexo feminino 33 (60\%), média de idade $55 \pm 18$ anos, cor branca 42 (76,3\%) e escolaridade inferior a nove anos de estudo 43 $(78 \%)$. Em relação às comorbidades clínicas, 44 (80\%) pacientes possuíam diagnóstico de HAS e $27(49,9 \%)$ de diabetes (Tabela 1$)$.

Tabela 1: Perfil sociodemográfico e clínico da amostra $(n=55)$. Porto Alegre, RS, Brasil. 2019.

\begin{tabular}{lc}
\hline Variáveis & $\mathbf{N}(\%)$ \\
\hline Sexo & $33(60)$ \\
Feminino & $55 \pm 8$ anos \\
Idade* & $28(50,9)$ \\
$<60$ anos & $27(49,1)$ \\
$\geq 60$ anos & \\
Raça/cor & $42(76,3)$ \\
Branca & $12(21,8)$ \\
Negra & $1(1,8)$ \\
Parda & \\
Escolaridade & $43(78)$ \\
$<9$ anos & $12(21,8)$ \\
$\geq 9$ anos & \\
Comorbidades clínicas & $44(80)$ \\
Hipertensão arterial sistêmica & $27(49,9)$ \\
Diabetes &
\end{tabular}

Dos 91 cateteres analisados, $47(51,6 \%)$ foram de curta e $44(48,3 \%)$ de longa permanência. A variação do tempo de permanência entre os cateteres de curta permanência foi de quatro a 190 dias, com mediana de 47 dias $(21,7-69,3)$. Em relação aos de longa permanência, esse tempo variou de 47 dias a quatro anos (1.486 dias) com mediana de 231 dias (95-676). O local de inserção mais prevalente dos cateteres de ambos os grupos foi em veia jugular direita $70(76,9 \%)$.

Os CVC de curta permanência foram retirados com maior frequência 32 (68\%) do que os de longa permanência $21(47,7 \%)$, dentre os principais motivos destacam-se, a troca do CVC de curta por um de longa permanência 17 (36\%), infecção ou suspeita de infecção sete $(15 \%)$ e início de diálise peritoneal quatro (9\%). Em relação aos de longa permanência os principais motivos de retirada foram infecção ou suspeita de infecção sete (16\%) e a presença de FAV funcionante seis $(13,6 \%)$. Ao término da coleta, seis $(12,7 \%)$ pacientes ainda estavam com cateter de curta permanência e $20(45,4 \%)$ de longa permanência, conforme apresentado na Tabela 2. Dentre o total de pacientes que utilizavam o CVC, apenas quatro $(6 \%)$ tinham registro em prontuário de esgotamento venoso para a confecção de FAV. 
Tempo de permanência e motivos de retirada do cateter venoso central de pacientes em hemodiálise ambulatorial

Tabela 2: Local de inserção e motivo de retirada do CVC ( $n$ = 91). Porto Alegre, RS, Brasil. 2019.

\begin{tabular}{|c|c|c|}
\hline Variáveis & $\begin{array}{l}\text { Cateter de curta permanência } \\
\qquad \mathrm{N}=47(\%)\end{array}$ & $\begin{array}{l}\text { Cateter de longa permanência } \\
\qquad \mathrm{N}=44(\%)\end{array}$ \\
\hline \multicolumn{3}{|l|}{ Local de inserção } \\
\hline Veia jugular direita & $39(82,9)$ & $31(70,4)$ \\
\hline Veia jugular esquerda & $6(12,7)$ & $8(18,1)$ \\
\hline Veias femorais direita/esquerda & $1(2,1)$ & $3(6,8)$ \\
\hline Veia subclávia direita & $1(2,1)$ & $2(4,5)$ \\
\hline \multicolumn{3}{|l|}{ Motivo de retirada do CVC } \\
\hline Troca por CVC de longa permanência & $17(36)$ & $0(0)$ \\
\hline Infecção/suspeita de infecção & $7(15)$ & $7(16)$ \\
\hline Iniciou diálise peritoneal & $4(9)$ & $1(2)$ \\
\hline Obstrução & $1(2)$ & $0(0)$ \\
\hline Baixo fluxo do CVC & $1(2)$ & $3(7)$ \\
\hline Fístula arteriovenosa funcionante & $1(2)$ & $6(14)$ \\
\hline Recuperação da função renal & $1(2)$ & $0(0)$ \\
\hline Óbito & $0(0)$ & $3(7)$ \\
\hline Extrusão cuff & $0(0)$ & $1(2)$ \\
\hline
\end{tabular}

\section{DISCUSSÃO}

Identificou-se variabilidade dos pacientes analisados, uma vez que a amostra abrangeu pacientes em tratamento dialítico por poucos dias até, aproximadamente, 12 anos. Nesse período, um único paciente chegou a dialisar 1.486 dias por CVC.

Foi identificado, dentre todos os pacientes que realizavam hemodiálise ambulatorial, que $40 \%$ usavam o CVC para o tratamento, quando segundo as recomendações científicas ${ }^{5}$ do Disease Outcomes Quality Initiative National Kidney Foundation (K/DOQI), esse percentual não deveria ultrapassar $20 \%$ do total de pacientes. Este dado sugere a importância de reavaliar tal prática, uma vez que os registros não justificam o motivo da não confecção de FAV.

No presente estudo verificou-se maior prevalência de pacientes do sexo feminino em tratamento dialítico, contrariando estudos que evidenciam maior frequência do sexo masculino, com $58 \%$ de homens ${ }^{8}$. Em relação à idade, segundo o censo nacional ${ }^{8}$, a maior parte dos pacientes tem mais de 60 anos, enquanto neste estudo apenas metade da amostra estudada tem essa correspondência.

As patologias associadas estudadas foram HAS e diabetes por serem as doenças de base de maior prevalência nas DRCs ${ }^{4}$. Estes achados vão ao encontro de dados do Censo Brasileiro de Nefrologia de 2018, em que a HAS e a diabetes apresentaram alta prevalência, sendo as principais causas da DRC no Brasili ${ }^{8}$.

O estudo identificou um tempo de permanência dos cateteres de curta permanência cinco vezes maior que o evidenciado pela literatura, que aponta período médio entre 8,5 e 9,3 dias ${ }^{9,10}$. Cabe ressaltar que a K/DOQI orienta, ainda, maximizar a probabilidade de início do tratamento com a FAV confeccionada e funcionante ${ }^{5}$. Segundo a Portaria 389/2014 do
Ministério da Saúde, os pacientes devem ser encaminhados para confecção da FAV ainda antes de iniciar a hemodiálise ${ }^{11}$.

O censo brasileiro de 2018 identificou que $77,4 \%$ dos pacientes com DRC realizavam hemodiálise por FAV e o restante por CVC de curta ou longa permanência. Além disso, no período de 2014 a 2018 , a prevalência de pacientes dialisando por cateteres apresentou redução de 5\% com relação aos de curta permanência e aumento de $138 \%$ nos de longa permanência, bem como no número de óbitos que passou de 25.187 em 2017 para 25.986 em $2018^{8}$.

Salienta-se que quatro pacientes estudados usaram CVC de longa permanência por não haver a possibilidade de outro acesso vascular, sendo este necessário para manutenção da terapia. Conforme o preconizado pela literatura ${ }^{12}$, estes são casos em que tais cateteres poderiam permanecer por muitos meses, devido a necessidade de cada indivíduo, funcionalidade e efetividade dos cuidados para evitar infecção. Este dado, em parte, explica o fato de alguns pacientes terem permanecido com acesso de longa permanência por longo período.

Os achados deste estudo podem estar relacionados ao fato de que um paciente, que estava em hemodiálise desde abril de 2000, apresentou elevado número de episódios infecciosos associado ao uso de CVC por impossibilidade de outra forma para dialisar. Este, excepcionalmente, fez uso de um cateter de longa permanência banhado com íons de prata, o que destoa da amostra geral, causando discrepância no tempo de hemodiálise com o mesmo cateter.

A maior prevalência do local de inserção dos cateteres foi em veia jugular direita. Esse dado vai ao encontro de pesquisas que indicam esta via de acesso como melhor opção de inserção pelo fato de reduzir o risco de estenose e comprometimento vascular que impossibilite a futura FAV devido ao 
acometimento dos vasos adjacentes ${ }^{8,10,13}$. Embora os vasos subclávios apresentem menor risco de infecção, há maior chance de outras complicações como: hemotórax, pneumotórax, perfuração e lesão de plexo braquial ${ }^{4,5,12,14}$. Os achados deste estudo evidenciaram a inserção do CVC nos vasos subclávios em apenas 2,3\% da amostra estudada, estando de acordo com o preconizado pela literatura que sugere priorizar os vasos jugulares ${ }^{7,14}$.

Um estudo nacional ${ }^{15}$ evidenciou que cateteres inseridos em veia femoral apresentaram maior índice de infecção, seguidos dos em veia jugular, corroborando com achados dessa pesquisa. Apesar da taxa de infecção deste estudo ser de $15,4 \%$ no total de cateteres implantados, quando analisados apenas os quatro inseridos em veia femoral, essa taxa passa a ser de $25 \%$. O acometimento infeccioso predispõe o paciente a um risco cinco vezes maior de apresentar intercorrências como febre e bacteremia ${ }^{9,10,12}$. Esses dados reforçam a necessidade da escolha de uma via de acesso com menor risco de intercorrências e infecção, sempre que possível, assim como de técnicas assépticas e capacitação das equipes envolvidas neste processo.

Os pacientes da amostra apresentaram um baixo número de infecção $(15,4 \%)$ quando comparados a outras pesquisas com percentuais próximos a $50 \%{ }^{6,9,10}$. Isso decorre, provavelmente, da técnica asséptica e rigorosa na forma como é feita a instalação e manipulação dos cateteres nesta instituição, seguindo recomendações de guidelines internacionais ${ }^{5,7}$ das capacitações que são realizadas de forma sistemática com os profissionais da saúde do serviço e na educação em saúde desenvolvida com os pacientes e familiares diariamente. Destaca-se ainda, que na instituição em estudo o manuseio dos cateteres é uma atividade exclusiva do profissional enfermeiro. Estes achados são corroborados com estudos que apontam a necessidade de padronização e uso de checklist para melhores desfechos, assim como a importância do enfermeiro orientar os pacientes para o autocuidado ${ }^{16,17}$. Outros autores apontam que os principais cuidados para prevenir infecção em paciente em diálise estão relacionados ao CVC, além de cuidados gerais voltados para as diretrizes de segurança do paciente ${ }^{18,19}$.

No presente estudo identificou-se como motivos para retirada do cateter a obstrução do CVC, extrusão do cuff, FAV funcionante, início em diálise peritoneal e troca de curta por de longa permanência. Tais dados são evidenciados na literatura que também inclui disfunção do cateter, recuperação da função renal, suspeita de infecção, óbito e alta ou transferência do paciente com o cateter ${ }^{10}$.

O principal motivo para retirar o CVC de curta permanência foi a troca por um de longa permanência, que por sua vez foi retirado por infecção ou, na presença de FAV funcionante após longo período. Estes resultados evidenciam uma prática controversa, visto que a recomendação é que a confecção da FAV seja realizada o mais precocemente possível e o acesso de escolha para iniciar hemodiálise ${ }^{5,20}$. A ausência de um acompanhamento ambulatorial nos estágios iniciais da DRC, somado com a dificuldade de encaminhamento para a confecção de FAV, após o início da terapia, favorece atraso na substituição da via de acesso para hemodiálise e expõe os pacientes a tempos excessivos com CVC. Entende-se que esses resultados merecem uma reflexão por parte da equipe multidisciplinar no sentido de pensar em estratégias para modificar essa prática.

As limitações se devem ao fato de ser um estudo retrospectivo com coleta de dados em prontuário eletrônico com restrições de algumas informações. Além disso, o período estudado, o tamanho amostral e a assimetria nos resultados não possibilitaram a realização de testes estatísticos para identificar correlações. No entanto, enfatiza-se a relevância dos dados encontrados, assim como a necessidade do seguimento deste estudo por meio de um acompanhamento prospectivo por um período de tempo maior, possibilitando ampliar o banco de dados e, assim, obter mais informações e um panorama desta realidade para análises mais robustas.

Reitera-se, também a necessidade do desenvolvimento de estudos sobre essa temática uma vez que a equipe de saúde está presente no cuidado do paciente em hemodiálise desde a chegada até a saída da unidade, evidenciando a importância de realizar uma assistência pautada nas melhores práticas visando a segurança do paciente e da equipe.

Os resultados revelam uma variabilidade na amostra, tanto em relação ao tempo de tratamento, quanto à permanência dos $\mathrm{CVC}$, evidenciando um uso excessivamente prolongado do mesmo. Diferente do que é recomendado pelo guideline K/DOQI, a principal causa de retirada do CVC não foi uso de FAV e sim outros oito motivos, prevalecendo a troca por outro CVC de longa permanência, embora a maioria dos pacientes da amostra não possuíam registro de esgotamento das possibilidades para confecção de FAV. Os índices de infecção encontrados não foram demasiadamente elevados, tendo em vista, o tempo de permanência destes cateteres. Entretanto, independentemente do tipo de CVC, existe uma exposição dos pacientes a complicações muitas vezes desnecessárias, sendo imperativo implementar providências visando minimizar os riscos.

Esses resultados apontam para a necessidade de promover uma revisão nos processos assistenciais, como priorizar a confecção de FAV precocemente e limitar o uso prolongado de CVC apenas para os casos de urgência e/ou impossibilidade de acesso permanente. Assim como, para a necessidade de 
desenvolver protocolos assistenciais visando qualificar o cuidado, preservar a segurança dos pacientes e equipe assistencial.

\section{Agradecimentos}

Agradecemos aos responsáveis pelo curso de especialização em nefrologia da Universidade Federal do Rio Grande do Sul (UFRGS) pela oportunidade da troca de conhecimento entre alunos e profissionais do Hospital de Clínicas de Porto Alegre (HCPA), e também aos profissionais de enfermagem da nefrologia que colaboraram com o desenvolvimento deste projeto de pesquisa.

\section{Conflitos de Interesse}

Os autores declaram não haver conflito de interesse.

\section{REFERÊNCIAS}

1. Lessa SRO, Bezerra JNM, Barbosa SMC, Luz GOA, Borba AKOT. Prevalência e fatores associados para a ocorrência de eventos adversos no serviço de hemodiálise. Texto Contexto Enferm. 2018;27(3):e3830017.

2. Sousa MRG, Silva AEBC, Bezerra ALQ, Freitas JS, Miasso Al. Eventos adversos em hemodiálise: relatos de profissionais de enfermagem. Rev Esc Enferm USP. 2013;47(1):76-83.

3. Schmidli J, Widmer MK, Basile C, Donato G, Gallieni M, Gibbons CP et al. Editor's choice - vascular access: 2018 clinical practice guidelines of the European Society for Vascular Surgery (ESVS). Eur J Vasc and Endovasc Surg. 2018;55(6):757-818.

4. Daugirdas JT, Blake PG, Ing TS. Manual de diálise. 5a ed. Rio de Janeiro: Guanabara Koogan; 2016.

5. Lok CE, Huber TS, Lee T, Shenoy S, Yevzlin AS, Abreo K, et al. KDOQI Clinical practice guideline for vascular access: 2019 update. Am J Kidney Dis. 2020;75:S1-S164.

6. Paiva PA, Paula BP, Santos MFF, Silveira BRM. Incidência de infecções da corrente sanguínea em pacientes nefropatas. Rev Aten Saude. 2018;16(55):72-80.

7. Frykholm P, Pikwer A, Hammarskjöld $F$, Larsson AT, Lindgren S, Lindwall R, et al. Clinical guidelines on central venous catheterisation. Acta Anaesthesiol Scand. 2014;58(5):508-24.
8. Sociedade Brasileira de Nefrologia. Censo 2018 [Internet]. São Paulo: Sociedade Brasileira de Nefrologia; 2018 [acesso 10 jul 2020].

Disponível em: https://www.sbn.org. $\mathrm{br} /$ profissional/censos-e-registros/ registro-brasileiro-de-dialise/.

9. Reisdorfer AS, Giugliani R, Gouveia VA, Santos EKM, Silva JJT. Infecção em acesso temporário para hemodiálise em pacientes com insuficiência renal crônica. Rev Pesqui Cuid Fundam. 2019;11(1):20-4.

10. Schwanke AA, Danski MTR, Pontes L, Kusma SZ, Lind J. Central venous catheter for hemodialysis: incidence of infection and risk factors. Rev Bras Enferm. 2018;71(3):1115-21.

11. Brasil. Ministério da Saúde. Portaria $n^{\circ} 389$, de 13 de março de 2014. Diário Oficial da União [Internet]. 2014 mar 14 [acesso 7 fev 2020]. Disponível em: http://bvsms.saude. gov.br/bvs/saudelegis/gm/2014/ prt0389_13_03_2014.html.

12. Zerati $A E$, Wolosker $N$, Luccia $N$ Puech-Leão P. Cateteres venosos totalmente implantáveis: histórico, técnica de implante e complicações. $J$ Vasc Bras. 2017;16(2):128-39.

13. Llapa-Rodríguez EO, Oliveira JKA, Melo FC, Silva GG, Mattos MCT, Macieira VP Jr. Inserção de cateter vascular central: adesão a bundle de prevenção de infecção. Rev Bras Enferm. 2019;72(3):810-6.

14. American Society of Anesthesiologists. Practice guidelines for central venous access 2020: an updated report by the
American Society of Anesthesiologists task force on central venous access. Anesthesiology. 2020;132(1):8-43.

15. Pinho NA, Silva GV, Pierin AMG. Prevalence and factors associated with chronic kidney disease among hospitalized patients in a university hospital in the city of São Paulo, SP, Brazil. J Bras Nefrol. 2015;37(1):91-7.

16. Duarte TAC, Alencar TD, Custódio N. Medidas preventivas nas práticas de inserção e manipulação de cateter de hemodiálise: estudo observacional. Rev Enferm Atual In Derme. 2017;81(19):70-5.

17. Coelho ECS, Pompeu HHFA, Ferreira IP, Souza AS, Castilho FNF, Santos VLC, et al. Conhecimento de pacientes em hemodiálise quanto ao autocuidado com cateter venoso central. REAS. 2018;11(2):e141.

18. Lira ALBC, Fernandes MICD, Silva FBBL, Fortes AV. Atención de enfermería a la prevención de infecciones en pacientes en hemodiálisis. Rev Cuba Enferm. 2018;34(1):182-96.

19. Ribeiro RC, Nobre RAM, Andrade EGS, Santos WL. O aumento das infecções relacionadas à hemodiálise por cateter venoso central. Rev Inic Cient Ext. 2018;1(5):432-8.

20. Brasil. Ministério da Saúde. diretrizes clínicas para o cuidado ao paciente com doença renal crônica - DRC no Sistema Único de Saúde. Brasília: Ministério da Saúde; 2014. 